\title{
Association of DLG5 and inflammatory bowel disease across populations
}

\author{
European Journal of Human Genetics (2006) 14, 259-260. doi:10.1038/sj.ejhg.5201516; published online 4 January 2006
}

In a recent report Daly et $a l^{1}$ replicated a previously reported association ${ }^{2}$ of the R30Q variant in the DLG5 gene with inflammatory bowel disease (IBD) in casecontrol samples from pooled Canadian and Italian populations. However, the groups were unable to replicate the findings in cases and controls from the United Kingdom. Similarly, Noble et $a l^{3}$ could not replicate the original association of R30Q to IBD reported by Stoll et al. ${ }^{2}$ Here, we present a combined analysis of the four data sets from cases and controls from Scotland, UK (excluding Scotland), pooled Canada/Italy and Germany. We used the metapackage of $\mathrm{R}$ software to perform a meta-analysis of all these studies. We found a substantial $\left(I^{2}=83.35 ; 95 \%\right.$ CI: 57.5-93.4\%) amount of heterogeneity among studies. This heterogeneity was statistically significant (Cochran's $Q$ statistic $=17.96 ; P=0.0004)$. A weak, but significant $(P=0.035)$, association of the R30Q variant and IBD was found under a fixed effect model but not under a random effects model. Under the fixed effect model, the R30Q variant was associated with an odds ratio (OR) of 1.19 (95\% confidence interval (CI): 1.01-1.39). The numbers used in the analysis are shown in Table 1.

We further investigated the source of the heterogeneity among studies by comparing the distribution of alleles in the different populations for both cases and controls. Overall there were significant differences in the distribution of alleles in the four populations (Fisher Exact test based on a 4 (populations) $\times 2$ (alleles) contingency table) for both cases $(P=0.022)$ and controls $(P=0.003)$. Table 2 shows the significance level of the six possible pairwise comparisons among the four populations using Fisher Exact test. It is worth noting that allele frequency differences among cases could be detected only for the UK/Germany comparison, whereas significant (or almost significant) differences could be detected among controls (again with the exception of the German/UK comparison). In summary, there are significant population allele frequency differences at the DLG5 gene (assuming the control sample is a representative sample of the general population) among the populations presented here. In fact allele frequencies at the DLG5 gene among IBD patients in different populations tend to be more similar than among controls. This is, in our view, inconsistent with the view of
Table 1 Number of alleles reported for each population in the original studies

\begin{tabular}{llrrcc}
\hline & Allele & Scotland & UK & Canada/Italy & Germany \\
\hline Controls & A & 67 & 96 & 24 & 93 \\
& G & 442 & 890 & 380 & 937 \\
IBD cases & A & 140 & 128 & 73 & 139 \\
& G & 1087 & 1250 & 591 & 911 \\
\hline
\end{tabular}

Table 2 Pairwise comparisons among populations

\begin{tabular}{lcccc}
\hline & Scotland & UK & Canada/Italy & Germany \\
\hline Scotland & & 0.0537 & 0.0003 & 0.0163 \\
UK & 0.0811 & & 0.0209 & 0.5935 \\
Canada/Italy & 0.8194 & 0.2346 & & 0.0543 \\
Germany & 0.1998 & 0.0025 & 0.1759 & \\
\hline
\end{tabular}

Above the diagonal are the $P$-values obtained from the comparisons of the control samples and below the diagonal are those from the comparisons of the case samples.

Daly et al $^{1}$ that heterogeneous replication results might be due to phenotypic differences among studies.

These population differences in allele frequency in both cases and particularly in controls are intriguing because they have relevance to pooling data from different populations in an attempt to identify novel loci contributing to complex disease and because genetic effects (measured as the allelic OR) will change with the population allele frequency (unless the locus mode of inheritance is multiplicative). This analysis suggests that pooling of genotyping data from studies in different populations might mask true but different effects among populations and that researchers will need to be very careful when pooling data from different populations.

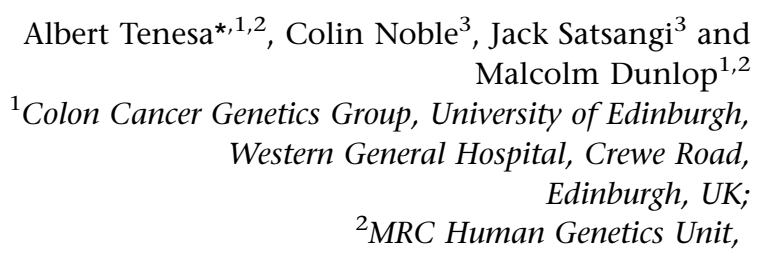


Western General Hospital, Crewe Road, Edinburgh, UK;

${ }^{3}$ Gastrointestinal Unit, Western General Hospital, Edinburgh, UK

${ }^{*}$ Correspondence: Dr A Tenesa, E-mail: albert.tenesa@ed.ac.uk.

\section{References}

1 Daly MJ, Pearce AV, Farwell L et al: Association of DLG5 R30Q variant with inflammatory bowel disease. Eur J Hum Genet 2005; 13: $835-839$.

2 Stoll M, Corneliussen B, Costello CM et al: Genetic variation in DLG5 is associated with inflammatory bowel disease. Nat Genet 2004; 36: 476-480.

3 Noble CL, Nimmo ER, Drummond $\mathrm{H}$ et al: DLG5 variants do not influence susceptibility to inflammatory bowel disease in the Scottish population. Gut 2005; 54: 1416-1420.

\section{Reply to Tenesa et al 'Association of DLG5 and inflammatory bowel disease across populations'}

European Journal of Human Genetics (2006) 14, 260-261. doi:10.1038/sj.ejhg.5201519; published online 4 January 2006

Tenesa et al raise an important issue regarding the involvement of DLG5 in IBD. Collectively, the heterogeneity of results at DLG5 is striking and we offer the following points for further discussion.

Point 1: In Daly et al (EJHG, 2005), we identified (and thus obviously concur with) the observation of heterogeneity among the studies we had aggregated - the overall combined data suggesting a small positive result and the fact that we confirmed a small positive effect in the additional family-based sample not included in this metaanalysis suggesting there is more than just noise here but additional studies will hopefully clarify. We are continuing to explore the source of this heterogeneity and feel that factors such as gender and ascertainment differences (which could lead to unexamined phenotypic differences) should be explored as proximal potential sources of the heterogeneity.

Point 2: We concur with the observation of heterogeneity of allele frequency across populations - it is not clear how unusual this observation is but several rarer coding polymorphisms and disease causing mutations have been noted to vary in frequency across European populations, including the most obvious comparison. The CARD15 mutations, which unequivocally confer that risks do appear to vary in frequency across European populations (for example in the paper by members of this correspondance in August 2004 Genes and Immunity).

Point 3: Here we have a slight difference in interpretation - a difference in population allele frequency does not have any directional or obligatory relationship to the risk conferred by that genotype. In this case, we have population samples that carry the allele in question between 6 and $12 \%$ or thereabouts - but whether the allele confers risk in each population is a completely independent question. While the estimated ORs could change very slightly, the heterogeneity observed is profound and ranges from showing quite significant association (Germany and Italy) to two that show none or a deficit of the risk allele among cases (UK and Scotland). A population frequency difference under any mode of inheritance cannot resolve these differences by itself although the population frequency difference could be an indirect marker for an ascertainment or population admixture difference that could be relevant.

The point that this commentary identifies that putting studies together is dangerous because different population frequencies can compromise accurate estimates since ORs can vary slightly is valid, and appropriate methods for evaluating association in the presence of heterogeneity should always be at hand. However, the implication that that is all that is needed to resolve the heterogeneity of these DLG5 studies is not supported by the analyses performed to date.

Further exploration of the source of heterogeneity at DLG5 is clearly warranted and Tenesa et al are right to highlight this fact - two studies (the German replication sample and Italy/Canada sample from Daly et al) show $P$-values of $\sim 0.001$ and two others from the UK and Scotland show no effect at all. As these are not simply cases of small sample discordancy but a highly significant discrepancy between studies that might have reasonably been considered to be addressing the same question, there is likely more to this story than has currently been discovered!

Best wishes 